\title{
Application Program Version or Upgrade Problem
}

National Cancer Institute

\section{Source}

National Cancer Institute. Application Program Version or Upgrade Problem. NCI

Thesaurus. Code C63304.

Problem associated with installing updates to a software system that affects a device performance or communication with another device. 